\title{
IMPROVEMENTS IN THE IRAS CALIBRATION BY SEPARATION OF THE DIFFERENT INFRARED SURFACE BRIGHTNESS COMPONENTS
}

\author{
D. J. M. Kester and P. R. Wesselius \\ Space Research, P.O. Box 800 \\ 9700 AV Groningen \\ The Netherlands
}

\author{
S. D. Price \\ $A F G L$ \\ Hanscom Air Force Base \\ Massachusetts 01731 USA
}

\begin{abstract}
A new calibration has been developed for GEISHA (Groningen Exportable Infrared System for High-Resolution Analysis) employing the zodiacal emission that is clearly present in each long scan. We have assumed, because of several other fundamental IRAS studies, that the zodiacal emission as a whole (ZEM) can be approximated by the emission of a tilted oblate ellipsoid emitting at a certain effective brightness. Besides this zodiacal emission, there is always a contribution of the galactic emission (GEM). As a standard candle, we take the sum of ZEM and GEM. We allow the electronic baseline to change linearly over a scan. We assume that the slow exponential decay that occurs will be sufficiently approximated by a straight line. The nonlinear behavior of the gain is corrected by a model developed in Groningen.
\end{abstract}

\section{INTRODUCTION}

The GEISHA system consists of a collection of software tools that allow an astronomer to create infrared sky maps from raw IRAS data. IRAS observed about $95 \%$ of the sky by a semi-overlapping scan technique. There are 62 detectors in the focal plane in four wavelength bands at 12, 25, 60, and $100 \mu \mathrm{m}$. (See IRAS Explanatory Supplement 1988, IRAS-ES hereafter.) The database of IRAS contains about 6000 scans running from one ecliptic pole to the other at a constant angle to the sun, the solar aspect angle.

\section{CALIBRATION}

One of the central items in the project is a calibration. The IRAS Processing and Analysis Center (IPAC) calibration has been created with special emphasis on correct and consistent fluxes of point sources. Their standard candle is the internal calibrator flash, which gives a response like a point source. The GEISHA calibration is tuned toward extended emission. The procedure for the GEISHA raw data calibration consists of autocalibration. For each detector scan, a set of calibration constants is extracted by fitting the lower envelopes of the detector scans to a standard candle, the parameters of which are derived from the same scans. Our standard candle is the zodiacal emission. Because of the rather simple spacial structure of this zodiacal emission, the emission can be used as a standard candle for calibration purposes.

The zodiacal emission as a whole is modeled by the emission of a tilted oblate ellipsoidal dust cloud. The density, temperature, and emissivity of the dust cloud have been combined into an effective emissivity. We assume that the geometry of the effective emissivity is similar in shape to the dust cloud: the effective emissivity is constant on ellipsoidal surfaces and it is a function of the size of the ellipsoids. For this function we introduce one extra parameter, which measures the relative mix between an effective emissivity that is constant throughout the cloud and one that decreases inversely proportional to the size of the ellipsoids, squared. 
The model has only five free parameters: the semi-major axis determining the outer edge of the zodiacal dust cloud, the ellipticity, the inclination with respect to the ecliptic, the angle of the ascending node, and the mix factor of the effective emissivity. The ZEM is a function of the solar aspect angle $\theta$, the longitude of the sun-referenced coordinate system $\psi$, and the position of the Earth, represented by the solar elongation $\lambda_{\odot}$.

Apart from the zodiacal emission, there is always a contribution of astronomical background (GEM); most of it is galactic emission. The astronomical background is so granulated that only a map of the whole sky can function as a model. GEM is added to the standard candle ZEM to provide a complete model of the brightness the detectors have observed.

The output, $O$, of a detector is a linearly changing electronic baseline plus the sum of ZEM and GEM multiplied by a time-dependent gain,

$$
O=a+b t+g(t)(Z E M(\psi, \theta, \lambda \odot)+G E M(\lambda, \beta)) .
$$

This complete detector model is fitted to all scans in the database, yielding a model of the zodiacal emission, a model for the behavior of the IR photodetectors on board IRAS, and a calibration of all scans. The calibration is still relative in the sense that it is not in absolute flux values, in $\mathrm{MJy}^{-1} \mathrm{r}^{-1}$.

\section{PRELIMINARY RESULTS}

We have been able to obtain a reasonable solution of the fit parameters in two of the IRAS bands: $12 \mu \mathrm{m}$ and $100 \mu \mathrm{m}$. The results for the zodiacal emission model are listed in Table 1 . The parameter representing the semi-major axis of the zodiacal emission model is somewhat larger than $1 \mathrm{AU}$, as was expected. However, it should be noted that the parameters for the semi-major axis and for the effective emissivity are not independent in this model. To a certain degree, one of these parameters can be fixed while the other adjusts itself. For example, choosing a value 0.0 for the effective emissivity at $100 \mu \mathrm{m}$ (i.e., an effective emissivity that is constant throughout the cloud) would force the semi-major axis to a somewhat larger value. The parameter for the ellipticity indicates that the cloud is quite flat-and flatter at larger wavelengths.

TABLE 1. Zodiacal Dust Cloud Parameters

\begin{tabular}{cccccc}
\hline \hline $\begin{array}{c}\text { Band } \\
(\mu \mathrm{m})\end{array}$ & $\begin{array}{c}\text { Semi-Major } \\
\text { Axis (AU) }\end{array}$ & Ellipticity & $\begin{array}{c}\text { Inclination } \\
\text { (degrees) }\end{array}$ & $\begin{array}{c}\text { Ascending Node } \\
\text { (degrees) }\end{array}$ & $\begin{array}{c}\text { Effective } \\
\text { Emissivity }\end{array}$ \\
\hline 12 & 1.58 & 0.94 & 2.0 & 52 & 0.99 \\
100 & 1.06 & 0.96 & 0.2 & 67 & 0.13 \\
\hline
\end{tabular}

${ }^{1}$ Values run from 0 to $1 ; 0$ indicates a constant effective emissivity and 1 indicates an $r^{-2}$ proportionality.

\section{REFERENCES}

IRAS Catalogs and Atlases, Explanatory Supplement Volume I 1988, ed. Beichman, C.A., Neugebauer, N., Habing, H.J., Clegg, P.E., Chester, T.J. NASA RP-1190, U.S. Government Printing Office, Washington, D.C. 
J.-L. Puget: Do you include changes of gain related to memory effects of the long wavelength detectors?

D. Kester: We plan to include a model for the memory effects at the larger wavelengths. This model was originally developed by Perault and Puget for the CPC instrument on board IRAS.

Ch. Leinert: Your $100 \mu \mathrm{m}$-model ellipsoid ends at $1.06 \mathrm{AU}$, slightly outside the earth's orbit. This is very different from what we know about interplanetary dust distribution. Could you comment on the reliability of the model result?

D. Kester: In the model the semi-major axis and the effective emissivity are not mutually independent. In the $100 \mu \mathrm{m}$ model presented here the effective emissivity is almost constant. In that case the outer edge moves in to $1.06 \mathrm{AU}$. I also tried the same data set on a model where the effective emissivity was fixed at $a^{-2}$; the outer edge showed a tendency to move outward to 20 AU or more. I have to investigate which choice of the effective emissivity yields a reasonable value for the semi-major axis. The mutual dependency reflects the fact that there is not enough information in the database to solve for both parameters in this very simple model. Concerning the zero point I can only say that IRAS was not an absolutely calibrated instrument. It has to be supplied with a model to set the zero point (IRAS Explanatory Supplement, p. VI-7).

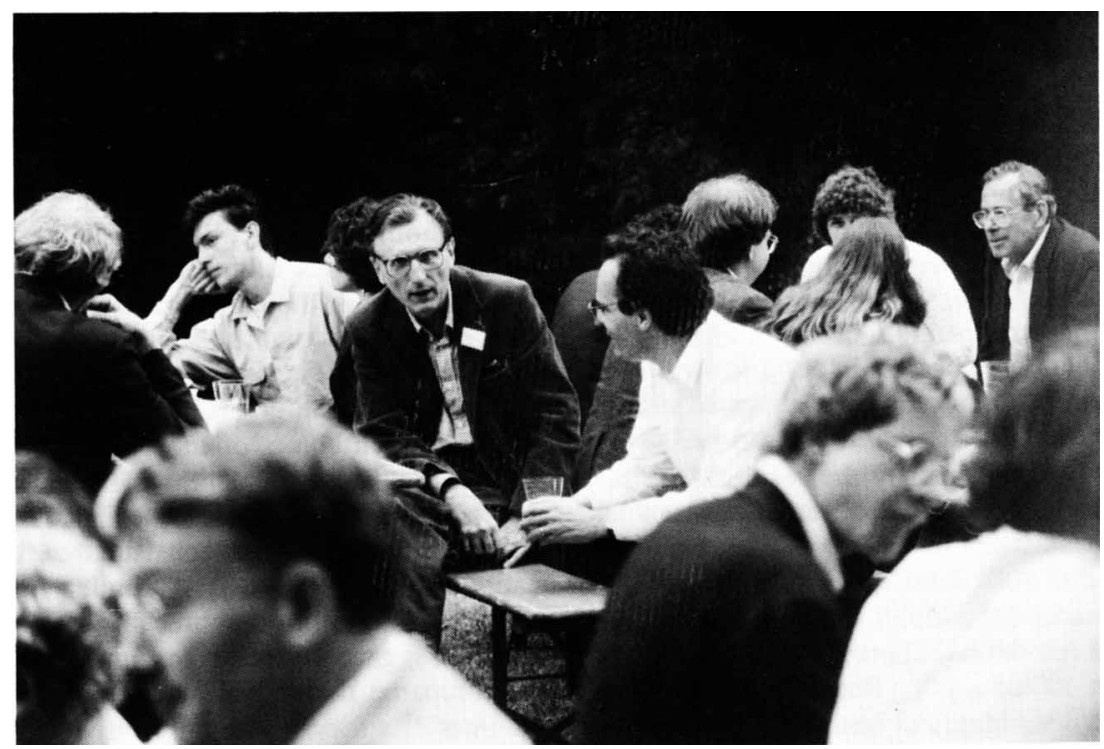

Center, discussing: Francesco Paresce and Jakob Staude; back right, Jim Peebles 